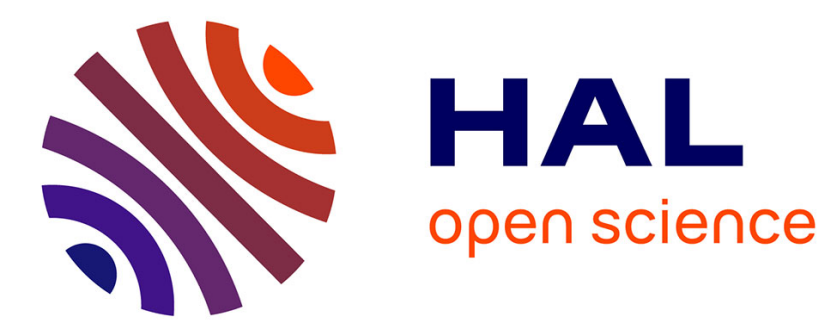

\title{
TMS reveals a direct influence of spinal projections from human SMAp on precise force production
}

Jonathan Entakli, Mireille B Bonnard, Sophie Chen, Eric Berton, Jozina B de Graaf

\section{- To cite this version:}

Jonathan Entakli, Mireille B Bonnard, Sophie Chen, Eric Berton, Jozina B de Graaf. TMS reveals a direct influence of spinal projections from human SMAp on precise force production. European Journal of Neuroscience, 2013, 39, pp.132 - 140. 10.1111/ejn.12392 . hal-01453467

\section{HAL Id: hal-01453467 \\ https://hal.science/hal-01453467}

Submitted on 22 Nov 2017

HAL is a multi-disciplinary open access archive for the deposit and dissemination of scientific research documents, whether they are published or not. The documents may come from teaching and research institutions in France or abroad, or from public or private research centers.
L'archive ouverte pluridisciplinaire HAL, est destinée au dépôt et à la diffusion de documents scientifiques de niveau recherche, publiés ou non, émanant des établissements d'enseignement et de recherche français ou étrangers, des laboratoires publics ou privés. 


\title{
TMS reveals a direct influence of spinal projections from human SMAp on precise force production
}

\author{
Jonathan Entakli, ${ }^{1}$ Mireille Bonnard, ${ }^{2}$ Sophie Chen, ${ }^{2}$ Eric Berton ${ }^{1}$ and Jozina B. De Graaf ${ }^{1}$ \\ ${ }^{1}$ Institute of Movement Sciences, Aix-Marseille University, CNRS, ISM UMR 7287, 163 avenue de Luminy, Marseille Cedex 09, \\ 13288, France \\ ${ }^{2}$ Aix-Marseille University, INSERM, INS UMR 1106, Marseille Cedex 05, France
}

Keywords: corticospinal tract, M1, precision grip, silent period

\begin{abstract}
The corticospinal (CS) system plays an important role in fine motor control, especially in precision grip tasks. Although the primary motor cortex (M1) is the main source of the CS projections, other projections have been found, especially from the supplementary motor area proper (SMAp). To study the characteristics of these CS projections from SMAp, we compared muscle responses of an intrinsic hand muscle (FDI) evoked by stimulation of human M1 and SMAp during an isometric static low-force control task. Subjects were instructed to maintain a small cursor on a target force curve by applying a pressure with their right precision grip on a force sensor. Neuronavigated transcranial magnetic stimulation was used to stimulate either left M1 or left SMAp with equal induced electric field values at the defined cortical targets. The results show that the SMAp stimulation evokes reproducible muscle responses with similar latencies and amplitudes as M1 stimulation, and with a clear and significant shorter silent period. These results suggest that (i) CS projections from human SMAp are as rapid and efficient as those from M1, (ii) CS projections from SMAp are directly involved in control of the excitability of spinal motoneurons and (iii) SMAp has a different intracortical inhibitory circuitry. We conclude that human SMAp and M1 both have direct influence on force production during fine manual motor tasks.
\end{abstract}

\section{Introduction}

Dexterity is based on the ability to independently and precisely control forces and movements of the fingers. Hand muscles for finger movements are steered by the lateral corticospinal system. In primates, where thumb and index finger can act in opposition, the main source of this corticospinal (CS) system can be traced to the primary motor area (M1), which has direct CS projections on motoneurons innervating intrinsic hand muscles (Maier et al., 1993; Porter \& Lemon, 1993; Armand et al., 1996). Studies have shown that lesions of the CS tract lead to a loss of fine motor control (Lawrence \& Kuypers, 1968a,b; Lemon \& Griffiths, 2005). Also, CS excitability in M1 increases with the precision of control (Hasegaw et al., 2001; Bonnard et al., 2007). These findings highlight the important role of CS projections in precise motor control.

Corticospinal projections from non-primary motor areas have also been found, especially from the supplementary motor area proper (SMAp) (Lemon et al., 1998; Nachev et al., 2008). In monkeys, these projections were found to be less dense and to have less excitatory effects than those from M1 (Palmer et al., 1981; Dum \& Strick, 1991). A recent human electroencephalography study showed a functional coupling between SMAp and hand muscles during isometric contractions requiring high precision (Chen et al., 2013),

Correspondence: Dr J. B. De Graaf, as above.

E-mail: jozina.de-graaf@univ-amu.fr

Received 15 April 2013, revised 13 September 2013, accepted 16 September 2013 strongly suggesting an implication of CS projections from SMAp. However, it gave no information about the transmission efficiency and velocity of the CS projections from SMAp. One way to study these characteristics is to use transcranial magnetic stimulation (TMS). Some TMS studies reported the presence of motor evoked potentials (MEPs) after SMAp stimulation (Teitti et al., 2008; Vaalto et al., 2011; Spieser et al., 2013). The MEPs showed latencies comparable with those obtained by M1 stimulation, suggesting the existence of CS projections from SMAp to motoneurons of hand muscles. However, in none of these studies were the induced electric fields equalized for both anatomical structures, making comparison of the evoked muscle responses between the structures difficult (Edgley et al., 1990). Moreover, some of these studies (Teitti et al., 2008; Vaalto et al., 2011) were done on relaxed subjects, i.e. not engaged in a specific motor task, so the CS projections may not have been active at the instant of the TMS. Therefore, a comparison of the MEP amplitudes obtained by stimulation of the two structures is not relevant and would not shed any light on the efficiency of the CS projections.

The present study aimed to compare the characteristics of CS projections from SMAp with those of M1, using neuronavigated TMS in subjects engaged in a precise manual force control task. As the depth of the two cortical structures is different (Picard \& Strick, 1996), in contrast to previous TMS studies we took particular care to equalize the induced electric field values at the anatomically defined stimulation targets. We quantified and compared MEP 
latencies, amplitudes and muscular silent periods in intrinsic hand muscle activity evoked by TMS on SMAp and M1.

\section{Materials and methods}

\section{Subjects}

Nine voluntary right-handed subjects (four females, five males; 25-46 years old) without known neurological pathology participated in this study. All subjects gave written informed consent. The experiment was approved by the local ethics committee (CPP Sud Méditerranée I) and was in accordance with the declaration of Helsinki. To avoid learning effects during the experiment, all subjects were familiarized with the force task a few days before the experiment to ensure stable performance. All subjects performed the task with the right hand.

\section{Experimental setup}

The subjects were comfortably seated in a clinical chair in front of a screen, with support for both hands and both arms. Their right arm and wrist in semi-pronation were immobilized horizontally in a plastic mould. This configuration allowed us to stabilize hand and finger positions, known to have an important influence on electromyographic (EMG) patterns. The left arm was maintained comfortably in a pillow and did not participate in the task (Fig. 1A). The subjects held a force sensor, fixed on a device, between the thumb and the index finger of the right hand. The screen displayed a small cursor as well as a force profile which moved with constant velocity from the right to the left on the screen. The horizontal position of the cursor was fixed in the middle of the screen while the vertical position was controlled by the subject.

\section{Force task protocol and experimental design}

The subjects were required to perform a visuomotor force tracking task. The cursor should be maintained on the force curve by pressure applied on a force sensor with the right precision grip. The cursor moved upwards with increasing force. The force exerted was measured by a constraint gauge fixed on the device. To have a less repetitive task and so to maintain the subject's attention, we used two cursor sizes both demanding a high level of precision (representing 0.2 and $0.4 \mathrm{~N}$ with respect to the force scale on the screen display).

There were two blocks of 40 trials. In each block, 20 trials were realized with the cursor of $0.2 \mathrm{~N}$ and 20 with the cursor of $0.4 \mathrm{~N}$, presented in a pseudo-random order. The force profile for each trial was composed of an increasing force ramp from 0 to $1.5 \mathrm{~N}$ in $4.5 \mathrm{~s}$ followed by a constant force level of $1.5 \mathrm{~N}$ lasting $5.5 \mathrm{~s}$ (Fig. 1B). The total duration of one trial was therefore $10 \mathrm{~s}$. At the end of each trial, the required force switched instantly from 1.5 to $0 \mathrm{~N}$ where it remained for $7 \mathrm{~s}$. This period of $7 \mathrm{~s}$ was a rest period during which subjects could release hand muscle activity. The total duration of one block was $12 \mathrm{~min}$.
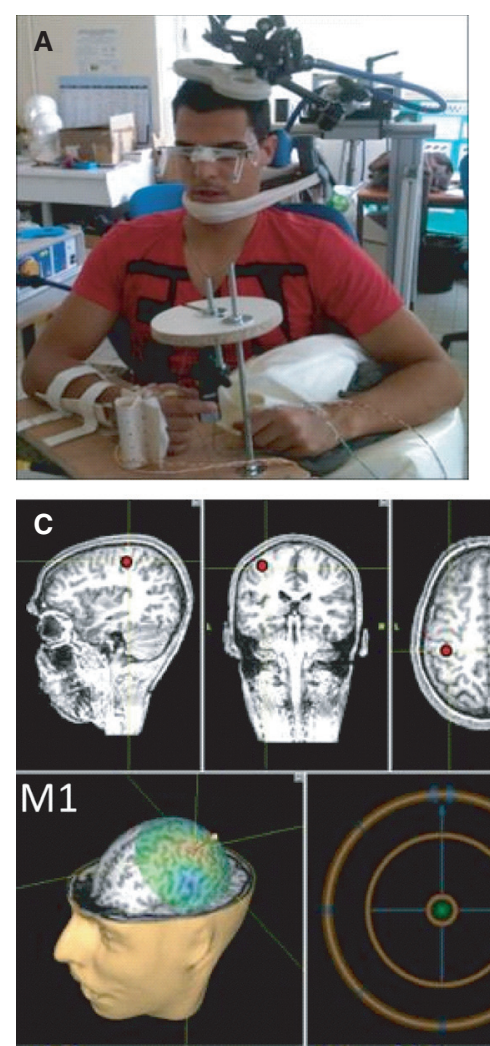

52
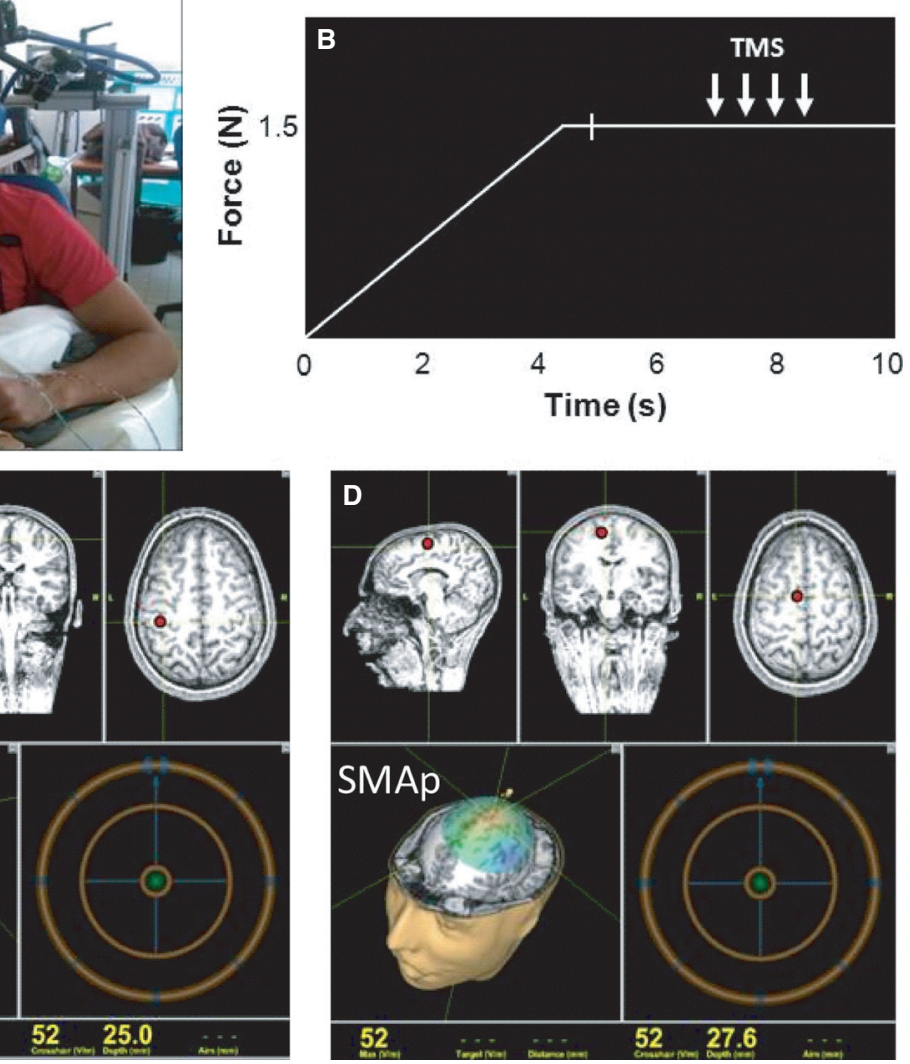

FIG. 1. Experimental setup. (A) TMS coil positioned over the stimulation site (here SMAp). The neuronavigation system can match the coil position with the anatomical image of the subject with help of markers placed on the glasses and the TMS coil. (B) Target force as a function of time (smallest cursor). The four possible instants of TMS are represented by white arrows. (C, D) 3D representation of the defined M1 (C) and SMAp (D) targets, represented by the red point on the anatomical image. 
Single-pulse TMS was delivered either over the hand area of the left motor cortex or over the hand area of the left supplementary motor area proper. During a block the cortical site of stimulation was unchanged. The TMS was triggered once every trial during the period of constant force production. To avoid anticipation of the stimulation, the TMS triggered at one of four different latencies $(2.5,3.0,3.5$ and $4.0 \mathrm{~s}$ after the beginning of the constant force production period; Fig. 1B). The subjects were informed that the TMS could induce small modifications of the cursor position on the screen, and were instructed to return the cursor on the force curve to continue the task whenever that happened. Throughout the experiment, the experimenter evaluated the subject's performance by visually checking on the screen the force generated by each subject. Furthermore, the experimenter monitored the muscle EMG level recorded during the task. During the period between every two blocks (i.e. during the placement of the TMS coil on the other site, see below), the subjects could rest to prevent fatigue.

\section{Neuronavigated TMS}

The stimulations were induced by a Magstim200 stimulator (Magstim Company, Whitland, UK). This stimulator can generate a maximum monophasic magnetic field of about $1.7 \mathrm{~T}$. It was connected to an eight-form coplanar coil with an extern loop diameter of $90 \mathrm{~mm}$. The coil was maintained in a desired position by using an articulated reel holder, located behind the chair in which the subject was seated. The coil could be placed, fixed and further stabilized by an experimenter. The stimulator was connected to a neuronavigation system (Navigated Brain Stimulation, Nexstim, Helsinki, Finland) that uses previously acquired anatomical magnetic resonance images of each individual subject to precisely guide the location and orientation of stimulation. The device locates the relative positions of the subject's head and of the TMS coil by means of an optical tracking system. In real time, this system assesses the distribution and strength of the intracranial electric field induced by the TMS pulse and projects it on the subject's magnetic resonance image. It also records the location and orientation of the coil at the instant of stimulation as well as the estimate of the electric field value on the stimulation targets.

M1 stimulation. The coil was positioned to stimulate the anterior bank of the left central sulcus at the level of the omega. This stimulation site corresponds to the right hand cortical representation of M1 (Rumeau et al., 1994; Yousry et al., 1997; Sastre-Janer et al., 1998). The coil handle was orientated backward, turned about $45^{\circ}$ clockwise with respect to the midline, to obtain an induced current directed forward, perpendicular to the central sulcus (Mills et al., 1992; Ziemann et al., 1999; Thielscher \& Kammer, 2002; Bonnard et al., 2007; Nardone et al., 2008; Bashir et al., 2013). For each subject, the target of stimulation was set at mid-distance between the bottom and the superior margin of the central sulcus. Figure 1C shows the M1 target for one individual subject. For each individual subject, the active motor threshold was defined as the minimal stimulation intensity necessary to produce an evoked muscle response of $50 \mu \mathrm{V}$ in five out of ten trials during, in our case, a constant isometric force output of $1.5 \mathrm{~N}$ (Rossini et al., 1988). The intensity value of the active motor threshold, averaged over all subjects, corresponded to $40.2 \pm 5.8 \%$ of maximal stimulator output (mean $\pm \mathrm{SD}$ ). The mean stimulation intensity was set at $110 \%$ of the active motor threshold. Across all subjects, this resulted in a mean of $43.7 \pm 5.7 \%$ of maximal stimulator output, the SD being due to inter-subject differences.
SMAp stimulation. In the sagittal plane, SMAp is defined as the region located between the anterior and posterior commissure (AC-PC), limited by the superior margin of the cingular gyrus and the superior margin of the cortex at the first frontal circumvolution (Matsunaga et al., 2005; Arai et al., 2011, 2012; Lu et al., 2012). For each subject, we defined the depth of stimulation at middistance between these two margins. In the sagittal plane, the cortical target was lateralized at the left side and positioned on the internal frontal face, in the juxta-cortical region. Figure 1D shows an example for a typical subject. The coil handle was initially orientated backward, turned about $15^{\circ}$ clockwise with respect to the midline. Its position was then optimized such that stimulation evoked the strongest MEP in FDI (Spieser et al., 2013). For each individual subject, we equalized the SMAp electric field at the target with that induced by the M1 stimulation at the defined M1 target. The mean stimulation intensity value was $55.7 \pm 5.8 \%$ of the maximal stimulator output. This was higher than that for the M1 stimulation as the electric field induced by the stimulation is dependent on its distance to the coil centre (Deng et al., 2013).

\section{Data acquisition}

Visualization of the force curve and the cursor was controlled by Labview software (acquisition card NI-6212). The recorded intrinsic hand muscles were the right and left FDI. This muscle allows the abduction of the index and assists the adductor pollicis in thumb adduction to realize the thumb/index opposition (precision grip). EMG signals were acquired by using small surface bipolar electrodes positioned on the muscle belly. The ground electrode was placed on the ulna's styloid process. The left FDI was recorded to detect eventual ipsilateral CS projections from SMAp. The produced force and the EMG data were acquired continuously on the same acquisition system (BrainAmp ExG, Brain Product Company, Gilching, Germany) at a sampling frequency of $2500 \mathrm{~Hz}$. EMG signals were filtered between 5 and $450 \mathrm{~Hz}$ before further processing. For each subject and stimulation, characteristics of the induced electric field (localization, maximal value) were recorded by the neuronavigation system. All signals were saved for off-line analysis.

\section{Data analysis}

\section{Selection of correct trials}

Behavioural and electrophysiological analyses were realized in Matlab (version 7.8). To verify the absence of difference in force output and background muscle activity between the two cursor sizes, we initially separated the trials as a function of cursor size. First, for each subject and trial, we verified that the cursor was positioned on the force curve during $1 \mathrm{~s}$ preceding stimulation. If this was not the case, the trial was removed from further analysis. Then to compare the produced grip force level and its variability between the two cursor sizes, we calculated the mean grip force as well as the SD during the $1 \mathrm{~s}$ preceding stimulation. Finally, because the quantity of EMG activity at the instant of stimulation (i.e. background) influences the motor response evoked by stimulation, we ensured that the EMG background was similar for each cursor size and each stimulation site (Aranyi et al., 1998; Hasegaw et al., 2001; Gagné $\&$ Schneider, 2007). So, for each trial, we averaged rectified EMG signals over a period of $100 \mathrm{~ms}$ before stimulation (Park \& Li, 2011). For each subject individually, we regrouped the background values per cortical stimulation site and cursor size, and we determined the common range of background values over all conditions. 
We then defined the maximal limit of this common range as the smallest maximal background value and the minimal limit as the largest minimal background value. If for a given trial the background value was not within this common range, the trial was removed from analysis (Schieppati et al., 1996).

\section{Global behavioural response}

As the stimulation intensity was set at $110 \%$ of active motor threshold, the TMS evoked a short increase of force production between thumb and index. This evoked force increase (i.e. the global behavioural response) is the net result of the evoked activity in all muscles involved in the force control task. To analyse the global behavioral response to the stimulation, for each correct trial, each cursor and each stimulation site we determined the peak of the force increase following the TMS pulse by calculating the difference between the peak force obtained after the TMS and the mean force level averaged over a period of $100 \mathrm{~ms}$ before stimulation.

\section{Spatial characteristics of the TMS}

For each subject we determined the Euclidean distance between the two anatomical stimulation targets (left SMAp and M1). Indeed, for each stimulation, the neuronavigation system provides threedimensional spatial coordinates of the defined targets as well as the value of the induced electric field at the defined target location. We also determined the value of the electric field induced at the defined target in M1 while stimulating SMAp (see Table 1).

\section{Evoked muscle responses}

The amplitude of the evoked potential was determined for each trial individually. This was defined as the absolute difference between the largest value and the smallest value of EMG signals during $50 \mathrm{~ms}$ following stimulation. Then, for each subject and each stimulation site, these individual MEP amplitude values were averaged over all trials, combining the two cursor sizes (for which statistics revealed no difference in force production and EMG background, see Results). MEP latency and the duration of the silent period (SP) were determined using the mean consecutive difference method (Garvey et al., 2001), illustrated in Fig. 3D and E. This method is complementary to that of visual inspection (Garvey et al., 2001; Säisänen et al., 2008) and commonly referred to as statistical process control with a confidence level usually fixed at $99.76 \%$ (equivalent to \pm 3SD of the mean rectified EMG) (Wheeler, 1993; Garvey et al., 2001). MEP latency was defined as the time from the instant of stimulation to the first of five successive samples above the high variation limit of the confidence interval (interval 1 in Fig. 3D and E). SP duration corresponded to the interval between the instant of stimulation and the first data point after the end of the MEP that rises above the lower variation limit if more than $50 \%$ of the data points in the following $5 \mathrm{~ms}$ window are also above the lower variation limit (interval 2 in Fig. 3D and E).

\section{Statistics}

The grip force production preceding stimulation was compared between the two cursor sizes with a Student's $t$-test for paired data. The EMG background and the global behavioural responses were compared between cursor sizes and stimulation sites by using a two-level ANOVA for repeated measures. As no difference was found between the two cursor sizes (see Results), a Student's $t$-test for paired data was used to compare the TMS characteristics (i.e. distance between targets, induced electric field values) and the muscle responses evoked by TMS. All statistical analyses were performed using Statistica (version 6) and the threshold of significance $(P=0.05)$ was maintained constant throughout analyses.

\section{Results \\ TMS characteristics}

Table 1 presents, for each subject and both stimulation sites, the stimulation intensity and electric fields induced at the level of the target at M1 and at SMAp. The mean depth of the target placed in M1 was $24.3 \pm 1.7 \mathrm{~mm}$. The mean electric field at the target location induced by stimulation of M1 was $53.0 \pm 6.0 \mathrm{~V} / \mathrm{m}$. The mean depth of the target placed in SMAp was $28.5 \pm 2.2 \mathrm{~mm}$. The mean induced electric field was $54.1 \pm 6.3 \mathrm{~V} / \mathrm{m}$ at the SMAp target location, i.e. similar to that at the M1 target induced by stimulation of M1 $(P>0.05)$, which was coherent with the experimental design. The mean Euclidian distance between the M1 and SMAp targets

TABLE 1. TMS settings

\begin{tabular}{|c|c|c|c|c|c|c|c|}
\hline \multirow[b]{2}{*}{ Subjects } & \multicolumn{3}{|l|}{ M1 } & \multicolumn{4}{|l|}{ SMAp } \\
\hline & Depth (mm) & $\begin{array}{l}\text { Stimulation } \\
\text { intensity }(\%)\end{array}$ & $\mathrm{EF}(\mathrm{V} / \mathrm{m})$ & Depth (mm) & $\begin{array}{l}\text { Stimulation } \\
\text { intensity }(\%)\end{array}$ & $\mathrm{EF}(\mathrm{V} / \mathrm{m})$ & $\mathrm{EF}$ at $\mathrm{M} 1(\mathrm{~V} / \mathrm{m})$ \\
\hline 1 & 23.5 & 40 & 54 & 27.4 & 50 & 55 & 41 \\
\hline 2 & 27.0 & 46 & 49 & 31.8 & 63 & 50 & 44 \\
\hline 3 & 23.3 & 38 & 49 & 28.9 & 47 & 49 & 40 \\
\hline 4 & 27.0 & 45 & 55 & 30.7 & 59 & 55 & 37 \\
\hline 5 & 25.1 & 57 & 64 & 26.1 & 65 & 65 & 47 \\
\hline 6 & 22.8 & 42 & 59 & 25.7 & 55 & 58 & 45 \\
\hline 7 & 22.8 & 45 & 53 & 27.2 & 53 & 60 & 50 \\
\hline 8 & 22.8 & 40 & 50 & 27.9 & 55 & 50 & 44 \\
\hline 9 & 24.7 & 40 & 44 & 30.7 & 54 & 45 & 38 \\
\hline Mean & 24.3 & 43.7 & 53.0 & 28.5 & 55.7 & 54.1 & 42.9 \\
\hline SD & 1.7 & 5.7 & 6.0 & 2.2 & 5.8 & 6.3 & 4.3 \\
\hline
\end{tabular}

Depth of the target location, stimulation intensity (\% of maximum stimulator output) and induced electric field values (EF) at the defined target for both stimulation sites and for each subject individually as well as the grand average values (Mean) and standard deviation (SD). For TMS on SMAp, the induced electric field value at the M1 target is also given. 
was $35.3 \pm 3.5 \mathrm{~mm}$ (SD being due to inter-subject variations in anatomy). At stimulation of SMAp, the electric field induced at the level of the M1 target was $42.9 \pm 4.3 \mathrm{~V} / \mathrm{m}$, which was significantly lower than that found during direct M1 stimulation $\left(t_{8}=5.5\right.$, $P<0.0005)$ and, moreover, below the active motor threshold (i.e. $48 \mathrm{~V} / \mathrm{m})$

\section{Selection of correct trials}

For both cursor sizes, all subjects maintained the cursor on the force curve during the whole second preceding the stimulation. So, no trials were removed based on the behavioral analysis. The grip force during this period was $1.44 \pm 0.03 \mathrm{~N}$ (grand average mean \pm grand average SD) for the $0.2 \mathrm{~N}$ cursor and $1.44 \pm 0.04 \mathrm{~N}$ for the $0.4 \mathrm{~N}$ cursor, showing the absence of a difference in grip force production between the two cursors $(P>0.05)$. After removing trials based on the EMG background, for each stimulation site and each cursor size, further analysis was realized for $16 \pm 3$ trials for the $0.2 \mathrm{~N}$ and $0.4 \mathrm{~N}$ cursors for $\mathrm{M} 1$, and $17 \pm 3$ trials for the $0.2 \mathrm{~N}$ cursor and $16 \pm 3$ trials for the $0.4 \mathrm{~N}$ cursor for SMAp (mean over subjects $\pm \mathrm{SD}$ ). A two-level ANOva for repeated measures showed neither global effects for stimulation site $\left(F_{1,8}=0.0006, P>0.05\right)$ and cursor size $\left(F_{1,8}=0.43, P>0.05\right)$ nor an interaction between stimulation site and cursor size $\left(F_{1,8}=4.0, P>0.05\right)$. So, no significant EMG background differences were found between the stimulation site and cursor sizes, which validated our procedure of selection. Therefore, differences in muscle responses evoked by TMS cannot be explained by differences in EMG values at the instant of stimulation.

\section{Global evoked grip force}

The global behavioural responses to the stimulation were similar for both cursor sizes and for both stimulation sites. Indeed, the twolevel ANOVA for repeated measures revealed neither global effects for stimulation site $\left(F_{1,8}=0.9, P>0.05\right)$ and cursor size $\left(F_{1,8}=0.57\right.$, $P>0.05)$, nor an interaction between stimulation site and cursor size $\left(F_{1,8}=0.03, P>0.05\right)$. As expected given the above mentioned absence of difference in produced grip force preceding the stimulation, no differences between the two cursor sizes were found. So, for each stimulation site, we gathered the trials for both cursor sizes and averaged the peak forces obtained for both cursor sizes. Figure 2 shows the time course of the global grip force, including the response to the TMS. No difference was found between the peak forces obtained for stimulation of M1 $(1.73 \pm 0.17 \mathrm{~N})$ and SMAp $(1.68 \pm 0.08 \mathrm{~N})\left(t_{8}=0.9, P>0.05\right)$.

\section{Muscle responses}

No EMG responses were obtained for left FDI, either for M1 stimulation or for SMAp stimulation. Therefore, the following results only concern right FDI. Figure 3 shows the high between-trial reproducibility, both of the coil location and orientation (Fig. 3A), and of the muscle responses evoked by the M1 (Fig. 3B) and SMAp stimulation (Fig. 3C), for a typical subject. A similar reproducibility was found for all other subjects.

Motor evoked potential latency, amplitude and duration of the SP, averaged over all subjects, are given in Table 2 for each stimulation site. Student's $t$-tests revealed no significant differences between both stimulation sites, either for MEP latency $\left(t_{8}=1.34, P>0.05\right)$ or for MEP amplitude $\left(t_{8}=0.54, P>0.05\right)$. However, the Student's $t$-test showed a significantly shorter SP duration for SMAp

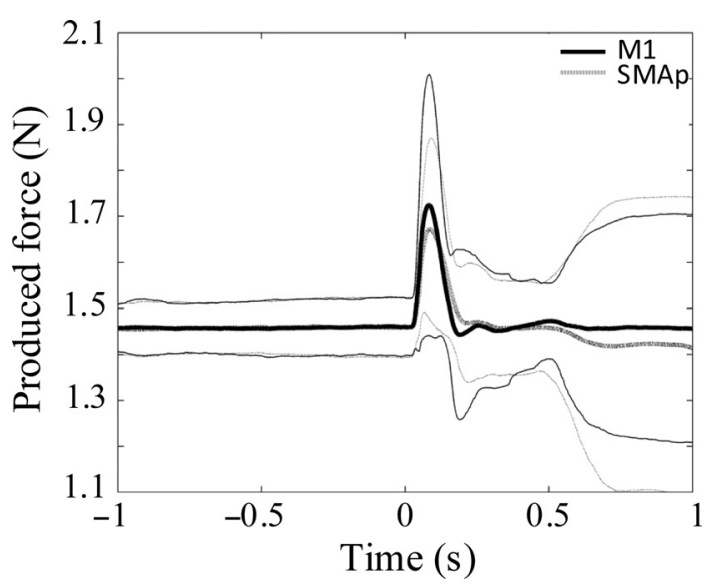

FIG. 2. Force output $( \pm$ SD) averaged over all subjects from $1 \mathrm{~s}$ before to $1 \mathrm{~s}$ after stimulation on M1 (bold black line) and on SMAp (bold dotted line). All traces are aligned at the instant of stimulation, so time 0 corresponds to the TMS pulse.

stimulation than for M1 stimulation $\left(t_{8}=2.8, P<0.02\right)$. So, resuming the main results, SMAp stimulation evoked MEPs on right FDI with similar latency and amplitude as M1 stimulation, but with a significantly shorter SP.

\section{Discussion}

The aim of the present study was to explore the characteristics of the CS projections from SMAp during a precise grip force control task. The subjects were instructed to perform a precise visuomotor force tracking task with the precision grip, known to maximally mobilize CS projections (Lemon et al., 1998). We compared intrinsic muscle responses evoked by TMS of left M1 and SMAp. Four main results were obtained. First, neither the stimulation of M1 nor that of SMAp evoked muscle responses of the left hand. Second, no differences were found in the global behavioural responses to the stimulation between both stimulation sites. Third, the latency and amplitude of the MEPs were similar for both stimulation sites. Finally, for similar induced electric fields at the defined targets, we found that the SP duration following SMAp stimulation was significantly shorter (25 ms on average) than that following M1 stimulation.

\section{Recruitment of CS projections from SMAp}

Obviously, to interpret these results correctly, it is important to assert that the electric field induced by SMAp stimulation does not co-activate M1 neurons. First, it is known that the strength of the magnetic field diminishes with the square of the distance to the coil centre (Deng et al., 2013). Due to the distance between the anatomical targets of SMAp and M1 (35 mm on average), the electric field value induced at the M1 target while stimulating SMAp (42.9 V/m) was indeed significantly lower than that induced by direct stimulation of M1 at $110 \%$ of the level of the active motor threshold (i.e. $53 \mathrm{~V} / \mathrm{m}$ ), and even lower than that at the active motor threshold $(48 \mathrm{~V} / \mathrm{m})$. As a consequence, it was significantly lower than the electric field required to obtain the reproducible MEPs we found when stimulating M1. Secondly, responses to TMS are extremely sensitive to the direction of the induced current (Bashir et al., 2013). A recent test (with subjects also included in the present study) showed that applying TMS at a site between M1 and SMAp 

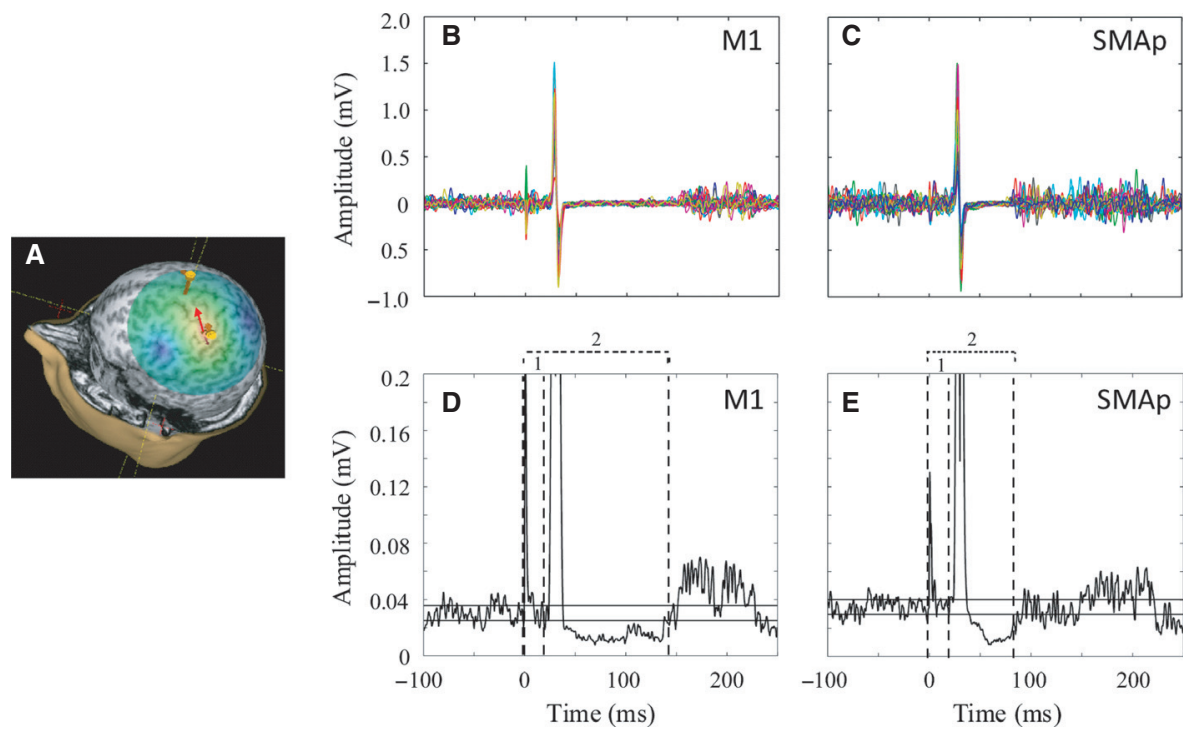

FIG. 3. Evoked muscle responses and the mean consecutive difference method for a typical subject. (A) Superposition of the markers (yellow dots) placed by the neuronavigation software on the anatomical image at each TMS for both M1 and SMAp. The electric field values induced by TMS at the M1 target are represented by colour (most intense at the target) and superimposed on the image. The red arrow indicates the orientation of the coil. (B, C) Superimposed EMG responses to TMS on M1 (B) and SMAp (C), recorded on right FDI during force tracking for all correct trials. Time 0 corresponds to the instant of the TMS pulse. Note the similarity of the evoked motor potentials for both stimulation sites. (D, E) Mean rectified EMG response to TMS on M1 (D) and SMAp (E), measured on right FDI during force tracking. The vertical dotted lines delimit the different analysed parts of the muscle responses (interval 1: Latency; interval 2: Silent period). The confidence interval is represented by the two horizontal black lines. Time 0 corresponds to the instant of the TMS pulse.

TABLE 2. Muscle responses

\begin{tabular}{lcc}
\hline & M1 & SMAp \\
\hline MEP latency (ms) & $23.1 \pm 1.9$ & $22.6 \pm 1.7$ \\
MEP amplitude (mV) & $1.4 \pm 0.9$ & $1.3 \pm 0.7$ \\
Silent period (ms) & $115.6 \pm 31.4$ & $87.4 \pm 20.1$
\end{tabular}

Grand average and standard deviation of MEP latency, MEP amplitude and SP for both M1 and SMAp stimulation.

but close to $M 1$, with the same intensity and coil orientation as those used for SMAp stimulation, only evoked very small muscle responses (see Spieser et al., 2013, for more details). Moreover, we did not find any evoked response on left FDI following stimulation of left SMAp. Although the left hand was relaxed (i.e. not engaged in the task), we should have evoked some responses if the electric field induced by TMS of left SMAp had activated CS neurons of right SMAp. So, despite the very small distance between left and right SMAp, the coil orientation for optimal stimulation of left SMAp (10-15 clockwise relative to the midline, i.e. almost parallel with the interhemispheric sulcus) was not adapted for stimulation of right SMAp, again showing the extreme sensitivity to coil orientation. Together, these results are coherent and indicate strongly that the electric field induced by stimulation of SMAp is not sufficient to efficiently stimulate M1. We can therefore conclude that the muscle responses to TMS on SMAp are not due to co-activation of M1.

The observed muscle responses evoked by stimulation over SMAp are not due to stimulation of M1 via direct projections from SMAp to M1, the latter projecting on the spinal cord. Indeed, the direct cortico-cortical connection between M1 and SMAp is known to transmit at a conduction velocity of about $10 \mathrm{~m} / \mathrm{s}$, and so this transmission would take around $6 \mathrm{~ms}$ (Civardi et al., 2001; Arai et al., 2012). If the muscle responses to SMAp stimulation were due to a massive recruitment of CS neurons of M1 following input from SMAp, we would have observed a difference of MEP latency of at least $6 \mathrm{~ms}$. This is obviously not the case as we found similar MEP latencies for M1 and SMAp stimulation, which is in accordance with other results reported in the literature (Teitti et al., 2008; Vaalto et al., 2011; Spieser et al., 2013). All arguments taken together, the muscle responses to stimulation of SMAp during our fine force production task are due to recruitment of corticospinal projections from this structure.

\section{CS projections from human SMAp as rapid and efficient as those from $M 1$}

Most studies on CS projections from non-primary motor areas have been realized on non-human primates (Maier et al., 2002; Boudrias et al., 2006; Lemon, 2008). In general, these studies have shown fewer CS neurons, lower conduction velocities and fewer CS connections to spinal motoneurons for projections coming from SMAp (12-19\% of fibres from pyramidal tract) than from M1. In addition, in non-human primates, CS projections from SMAp are found to have less common excitatory effects than for M1, and the excitatory post-synaptic potential is much smaller (Lemon et al., 2002; Maier et al., 2002). Given these findings, one would naturally expect slower and smaller MEPs following TMS of SMAp than following stimulation of M1. It therefore seems rather surprising that we found similar MEP latencies and amplitudes for both stimulated sites.

Similar latencies have already been shown in other recent studies on TMS of human non-primary motor areas (Teitti et al., 2008; Vaalto et al., 2011; Spieser et al., 2013), but in the two latter studies the authors found different MEP amplitudes. In fact, they found larger amplitudes for non-primary motor areas than for M1, which is unexpected given the above-mentioned results obtained in nonhuman primates. It is, however, difficult to compare MEP 
amplitudes between different cortical stimulation sites. The three mentioned TMS studies did not equalize the induced electric fields at the targets, thereby introducing differences in stimulated brain volume between different stimulation sites. In the present study, we made the choice to define the target at mid-height of the cortical sulcus (see Methods) and adapted the stimulation intensity to equalize the induced electric field at both targets. In these conditions, we found similar amplitudes of muscle responses to TMS of M1 and of SMAp.

The present results are based on the responses of only one of the muscles involved in the motor task, so one might claim that the similarity in MEPs obtained by stimulation of the two cortical sites is obtained by 'chance' and that different results could be obtained for other muscles involved in the task. However, we found similar global behavioural responses to the stimulation for both stimulation sites, showing that the muscle responses of all muscles involved in the force control task were similar following stimulation of both cortical sites. Altogether, our results suggest similar excitability of CS neurons in human M1 and SMAp during a precise force control task, with, moreover, CS projections from human SMAp as rapid and as efficient as those from M1.

\section{SP reveals a direct influence of SMAp spinal projections on force production}

According to the literature, the SP has two origins. The first is related to spinal mechanisms and concerns only the first $50-60 \mathrm{~ms}$ following the instant of stimulation (Inghilleri et al., 1993). The second origin is widely accepted to be related to cortical mechanism and concerns the rest of the SP until the uninterrupted recovery of muscle activity ( $\mathrm{SP}>60 \mathrm{~ms}$ ) (Inghilleri et al., 1993). Given that we found SP durations of about $115 \mathrm{~ms}$ following TMS of M1 and about $90 \mathrm{~ms}$ following stimulation of SMAp, the difference in SP duration between the two cortical structures is most probably related to cortical mechanisms. The shorter SP following SMAp stimulation relative to M1 stimulation might reflect a less developed cortical inhibition network in SMAp, thereby facilitating the earlier reactivation of neurons in this cortical structure with respect to M1. This hypothesis could be tested with the technique of paired-pulse TMS (Kujirai et al., 1993).

The fact that SMAp stimulation induces a silent period allows us to hypothesize about the spinal targets of SMAp spinal projections. It has been suggested that in non-human primates, SMAp can act in parallel and independently of M1 during a motor task (Brinkman \& Porter, 1979; Macpherson et al., 1982; Maier et al., 2002). Also, axons of neurons recruited in M1 and SMAp use the same descending CS tract (Kouchtir-Devanne et al., 2012), and part of these CS projections might converge to the same alpha motoneurons (Lemon et al., 2002; Maier et al., 2002). This implies that fibres from M1 and SMAp can control the same muscle fibres. So, the deactivation of the descending fibres from SMAp by intra-cortical inhibition causes lower input to those motoneurons that also receive fibres from M1. If the input from M1 alone is not enough to exceed the threshold of the concerned motoneurons, the targeted muscle fibres are silenced, causing the observed SP in the muscle responses. This hypothesis requires of course that a sufficient number of spinal motoneurons are innervated by both SMAp and M1 descending fibres (with similar total weights for both inputs), which remains to be seen for the human corticospinal tract. However, whatever the underlying mechanism, our results clearly suggest that SMAp and M1 both have direct influence on force production during fine manual motor tasks.

\section{Evolution of the CS tract in primates}

One reason for the difference in efficiency of corticospinal (motoneuronal) projections between non-human and human primates might be that human dexterity is a highly developed function. At the phylogenetic level, the development of the thumb-index opposition has been found to be related to the evolution of the CS tract, especially with the apparition of direct cortico-motoneuronal connections (Lemon et al., 1995; Nakajima et al., 2000). The similar latencies of the MEPs following TMS of M1 and SMAp strongly support the hypothesis that SMAp is also monosynaptically connected to motoneurons of the spinal cord, partly on the same motoneurons, as argued above. A recent human electroencephalographic study showed corticomuscular coherence between SMAp and two intrinsic hand muscles involved in the same force control task as in the present study (Chen et al., 2013). The corticomuscular coherence over the SMAp region was strong, reflecting the important involvement of CS projections from SMAp during this task. So, our results suggest that due to the more developed role of manual dexterity in human daily life, corticomotoneuronal projections from non-primary motor areas, especially SMAp, have gained in efficiency.

The exact role of spinal projections from SMAp cannot be inferred from the present study. It has been suggested that SMAp is involved in control of the excitability of spinal motoneurons (Macpherson et al., 1982) and in precise manual force control (Smith, 1979; Ikeda et al., 1992; Kuhtz-Buscbeck et al., 2001; Bonnard et al., 2007), which are propositions in line with our hypothesis. It has also been found that unilateral ablation of SMAp in non-human primates leads to a loss of coordination in the contralateral precision grip, i.e. the monkeys lose the capability of picking-up food between thumb and index finger (Brinkman, 1984), which suggests that the descending pathway from SMAp might play a role in specifically controlling thumb opposition to the other fingers. Finally, also using TMS of SMAp, Spieser et al. (2013) suggested that SMAp plays a role in anticipatory processes during expectation of perturbation. Although the only perturbation that was expected in our experiment was the short TMS-evoked grip force increase that the subjects were instructed to ignore, our results might suggest that SMAp has a direct influence on motoneuron excitability to control the grip force reaction to eventual perturbations.

\section{Conclusion}

The present study showed that, during a precise force control task, TMS on SMAp evokes motor potentials (MEPs) on intrinsic hand muscles similar to those evoked by TMS on M1. For equal induced electric field values at the cortical targets, the latency and the amplitude of the MEPs were equivalent, suggesting that CS projections from human SMAp are as rapid and efficient as those from M1. As descending fibres from M1 are known to project directly onto spinal motoneurons innervating intrinsic hand muscles, the similar latencies strongly suggest that human SMAp also directly project onto spinal motoneurons. The SP was found to be shorter following SMAp stimulation than following M1 stimulation, which probably reflects differences between SMAp and M1 in local intracortical inhibitory projections. The fact that the SMAp stimulation can induce a muscular SP despite ongoing activity of M1 strongly suggests that the CS projections from SMAp are directly involved in control of the excitability of spinal motoneurons. In conclusion, the present results strongly suggest that, in humans, SMAp and M1 both have 
direct and effective influence on force production during fine manual motor tasks.

\section{Acknowledgements}

We thank T. Coyle for correcting the English language and an anonymous reviewer for suggested improvements to a previous version of the manuscript. This study was supported by the Centre National de la Recherche Scientifique.

\section{Abbreviations}

CS, corticospinal; EMG, electromyography; FDI, first dorsal interosseous; MEP, motor evoked potential; M1, primary motor cortex; SMAp, supplementary motor area proper; SP, silent period; TMS, transcranial magnetic stimulation.

\section{References}

Arai, N., Müller-Dahlhaus, F., Murakami, T., Bliem, B., Lu, M.K., Ugawa, Y. \& Ziemann, U. (2011) State-dependent and timing-dependent bidirectional associative plasticity in the human SMA-M1 network. J. Neurosci., 31, 15376-15383.

Arai, N., Lu, M.K., Ugawa, Y. \& Ziemann, U. (2012) Effective connectivity between human supplementary motor area and primary motor cortex: a paired-coil TMS study. Exp. Brain Res., 220, 78-87.

Aranyi, Z., Mathis, J., Hess, C. \& Rosler, K.M. (1998) Task-dependent facilitation of motor evoked potentials during dynamic and steady muscle contractions. Muscle Nerve, 21, 1309-1316.

Armand, J., Olivier, E., Edgley, S.A. \& Lemon, R. (1996) The structure and function of the developing corticospinal tract. Some key issues. In Wing, A., Haggard, P. \& Flanagan, J.R. (Eds), Hand and Brain: The Neurophysiology and Psychology of Hand Movements. Academic Press, San Diego, pp. $125-145$.

Bashir, S., Perez, J.M., Horvath, J.C. \& Pascual-Leone, A. (2013) Differentiation of motor cortical representation of hand muscles by navigated mapping of optimal TMS current directions in healthy subjects. J. Clin. Neurophysiol., 30, 390-395.

Bonnard, M., Galléa, C., De Graaf, J.B. \& Pailhous, J. (2007) Corticospinal control of the thumb-index grip depends on precision of force control: a transcranial magnetic stimulation and functional magnetic resonance imagery study in humans. Eur. J. Neurosci., 25, 872-880.

Boudrias, M.H., Belhaj-Saö̈f, A., Park, M.C. \& Cheney, D. (2006) Contrasting properties of motor output from the supplementary motor area and primary motor cortex in rhesus macaques. Cereb. Cortex, 16, 632-638.

Brinkman, C. (1984) Supplementary motor area of the monkey's cerebral cortex: short- and long-term deficits after unilateral ablation and the effects of subsequent callosal section. J. Neurosci., 4, 918-929.

Brinkman, C. \& Porter, R. (1979) Supplementary motor area in monkey: activity of neurons during performance of a learned motor task. J. Neurophysiol., 42, 681-709.

Chen, S., Entakli, J., Bonnard, M., Berton, E. \& De Graaf, J.B. (2013) Functional corticospinal projections from human supplementary motor area revealed by corticomuscular coherence during precise grip force control. PLOS ONE, 8, e60291.

Civardi, C., Cantello, R., Asselman, P. \& Rothwell, J.C. (2001) Transcranial magnetic stimulation can be used to test connections to primary motor areas from frontal and medial cortex in humans. Neurolmage, 14, 1444-1453

Deng, Z.D., Lisanby, S.H. \& Peterchev, A.V. (2013) Electric field depthfocality tradeoff in transcranial magnetic stimulation: simulation comparison of 50 coil designs. Brain Stimul., 6, 1-13.

Dum, R.P. \& Strick, P.L. (1991) The origin of corticospinal projections from the premotor areas frontal lobe. J. Neurosci., 11, 667-689.

Edgley, S.A., Eyre, J.A., Lemon, R.N. \& Miller, S. (1990) Excitation of the corticospinal tract by electromagnetic and electrical stimulation of the scalp in the macaque monkey. J. Physiol., 425, 301-320.

Gagné, M. \& Schneider, C. (2007) Dynamic changes in corticospinal control of precision grip during wrist movements. Brain Res., 1164, 32-43.

Garvey, M.A., Ziemann, U., Becker, D.A. \& Bartko, J.J. (2001) New graphical method to measure silent periods evoked by transcranial magnetic stimulation. Clin. Neurophysiol., 112, 1451-1460.
Hasegaw, Y., Kasai, T., Kinoshita, H. \& Yahagi, S. (2001) Modulation of a motor evoked response to transcranial magnetic stimulation by the activity level of the first dorsal interosseous muscle in humans when grasping a stationary object with different grip widths. Neurosci. Lett., 299, 1-4.

Ikeda, A., Lüders, H.O., Burgess, R.C. \& Shibasaki, H. (1992) Movementrelated potentials recorded from supplementary motor area: role of supplementary motor area in voluntary movements. Brain, 115, 1017-1043.

Inghilleri, M., Berardelli, A., Gruccu, G. \& Manfredi, M. (1993) Silent period evoked by transcranial stimulation of the human cortex and cervicomedullary junction. J. Physiol., 466, 521-534.

Kouchtir-Devanne, N., Capaday, C., Cassim, F., Derambure, P. \& Devanne, H. (2012) Task-dependent changes of motor cortical network excitability during precision grip compared to isolated finger contraction. J. Neurophysiol., 107, 1522-1529.

Kuhtz-Buscbeck, J.P., Ehrsson, H.H. \& Forssberg, H. (2001) Human brain activity in the control of fine static precision grip forces: an fMRI study Eur. J. Neurosci., 14, 382-390.

Kujirai, T., Caramia, M.D., Rothwell, J.C., Day, B.L., Thompson, P.D., Ferbert, A., Wroe, S., Asselman, P. \& Marsden, C.D. (1993) Corticocortical inhibition in human motor cortex. J. Neurophysiol., 471, 501-519.

Lawrence, D.G. \& Kuypers, H.G.J.M. (1968a) The functional organization of the motor system in the monkey. I. The effects of bilateral pyramidal lesions. Brain, 91, 1-14.

Lawrence, D.G. \& Kuypers, H.G.J.M. (1968b) The functional organization of the motor system in the monkey. II. The effects of lesions of the descending brain-stem pathway. Brain, 91, 15-36.

Lemon, R.N. (2008) Descending pathways in motor control. Аnпи. Rev. Neurosci., 31, 195-218.

Lemon, R.N. \& Griffiths, J. (2005) Comparing the function of the corticospinal system in different species: organizational differences for motor specialization? Muscle Nerve, 32, 261-279.

Lemon, R.N., Johansson, R.S. \& Westling, G. (1995) Corticospinal control during reach, grasp, and precision lift in man. J. Neurosci., 15, 6145-6156.

Lemon, R.N., Baker, S.N., Davis, J.A., Kirkwood, P.A., Maier, M.A. \& Yang, H.S. (1998) The importance of the cortico-motoneuronal system for control of grasp. Novart. Fdn. Symp., 218, 202-218.

Lemon, R.N., Maier, M.A., Armand, J., Kirkwood, P.A. \& Yang, H.W (2002) Functional differences in corticospinal projections from macaque primary motor cortex and supplementary motor area. Adv. Exp. Med. Biol., 508, 425-434.

Lu, M.K., Arai, N., Tsai, C.H. \& Ziemann, U. (2012) Movement related cortical potentials of cued versus self-initiated movements: double dissociated modulation by dorsal premotor cortex versus supplementary motor area rTMS. Hum. Brain Mapp., 33, 824-839.

Macpherson, J.M., Wiesendanger, M., Marangoz, C. \& Miles, T.S. (1982) Corticospinal neurons of the supplementary motor area of the monkey. Exp. Brain Res., 48, 81-88.

Maier, M.A., Bennett, K.M., Hepp-Reymond, M.C. \& Lemon, R.N. (1993) Contribution of the monkey corticomotoneuronal system to the control of force in precision grip. J. Neurophysiol., 69, 772-785.

Maier, M.A., Armand, J., Kirkwood, P.A., Yang, H.-W., Davis, J.N. \& Lemon, R.N. (2002) Differences in the corticospinal projection from primary motor cortex and supplementary motor area to macaque upper limb motoneurons: an anatomical and electrophysiological study. Cereb. Cortex, 12, 281-296.

Matsunaga, K., Maruyama, A., Fujiwara, T., Nakanishi, R., Tsuji, S. \& Rothwell, J.C. (2005) Increased corticospinal excitability after $5 \mathrm{~Hz}$ rTMS over the human supplementary motor area. J. Physiol., 562, 295-306.

Mills, K.R., Boniface, S.J. \& Schubert, M. (1992) Magnetic brain stimulation with a double coil: the importance of coil orientation. Electroen. Clin. Neuro., 85, 17-21.

Nachev, P., Kennard, C. \& Husain, M. (2008) Functional role of the supplementary and pre-supplementary motor areas. Nat. Rev. Neurosci., 9 856-869.

Nakajima, K., Maier, M.A., Kirkwood, P.A. \& Lemon, R.N. (2000) Striking differences in transmission of corticospinal excitation to upper limb motoneurons in two primate species. J. Neurophysiol., 84, 1-12.

Nardone, R., Venturi, A., Ausserer, H., Ladurner, G. \& Tezzon, F. (2008) Cortical silent period following TMS in a patient with supplementary sensorimotor area seizures. Exp. Brain Res., 184, 439-443.

Palmer, C., Schmidt, E.M. \& McIntosh, J.S. (1981) Corticospinal and corticorubral projections from the supplementary motor area in the monkey. Brain Res., 209, 305-314.

Park, W.-H. \& Li, S. (2011) No graded responses of finger muscles to TMS during motor imagery of isometric finger forces. Neurosci. Lett., 494, 255-259. 
140 J. Entakli et al.

Picard, N. \& Strick, P.L. (1996) Motor areas of the medial wall: a review of their location and functional activation. Cereb. Cortex, 6, 342-353.

Porter, R. \& Lemon, R.N. (1993) Corticospinal Function and Voluntary Movement. Physiological Society Monograph. Oxford University Press, Oxford.

Rossini, P.M., Zarola, F., Stalberg, E. \& Caramia, M. (1988) Pre-movement facilitation of motor-evoked potentials in man during transcranial stimulation of the central motor pathways. Brain Res., 458, 20-30.

Rumeau, C., Tzourio, N., Murayama, N., Peretti-Viton, P., Levrier, O., Joliot, M., Mazoyer, B. \& Salamon, G. (1994) Location of hand function in the sensorimotor cortex: MR and functional correlation. Am. J. Neuroradiol., 15, 567-572.

Säisänen, L., Pirinen, E., Teitti, S., Könönen, M., Julkunen, P., Määttä, S. \& Karhu, J. (2008) Factors influencing cortical silent period: optimized stimulus location, intensity and muscle contraction. J. Neurosci. Meth., 169, 231-238.

Sastre-Janer, F.A., Regis, J., Belin, P., Mangin, J.F., Dormont, D., Masure, M.C., Remy, P., Frouin, V. \& Samson, Y. (1998) Three-dimensional reconstruction of the human central sulcus reveals a morphological correlate of the hand area. Cereb. Cortex, 8, 641-647.

Schieppati, M., Trompetto, C. \& Abbruzzese, G. (1996) Selective facilitation of responses to cortical stimulation of proximal and distal arm muscles by precision tasks in man. J. Physiol., 491, 551-562.

Smith, A.M. (1979) The activity of supplementary motor area neurons during a maintained precision grip. Brain Res., 172, 315-327.
Spieser, L., Aubert, S. \& Bonnard, M. (2013) Involvement of SMAp in the intention-related long latency stretch reflex modulation: a TMS study. Neuroscience, 246, 329-341.

Teitti, S., Määttä, S., Säisänen, L., Könönen, M., Vanninen, R., Hannula, H., Mervaala, E. \& Karhu, J. (2008) Non-primary motor areas in the human frontal lobe are connected directly to hand muscles. Neurolmage, 40, 1243-1250

Thielscher, A. \& Kammer, T. (2002) Linking physics with physiology in TMS: a sphere field model to determine the cortical stimulation site in TMS. Neurolmage, 17, 1117-1130.

Vaalto, S., Säisänen, L., Könönen, M., Julkunen, P., Hukkanen, T., Määttä, S. \& Karhu, J. (2011) Corticospinal output and cortical excitation-inhibition balance in distal hand muscle representation in nonprimary motor area. Hum. Brain Mapp., 32, 1692-1703.

Wheeler, D.J. (1993) Understanding Variation: The Key to Managing Chaos. TN, SPC Press, Knoxville.

Yousry, T.A., Schmid, U.D., Alkadhi, H., Schmidt, D., Peraud, A., Buettner, A. \& Winkler, P. (1997) Localization of the motor hand area to a knob on the precentral gyrus. A new landmark. Brain, 120, 141-157.

Ziemann, U., Ishii, K., Borgheresi, A., Yaseen, Z., Battaglia, F., Hallett, M., Cincotta, M. \& Wassermann, E.M. (1999) Dissociation of the pathways mediating ipsilateral and contralateral motor-evoked potentials in human hand and arm muscles. J. Physiol., 518, 895-906. 